\title{
Complications in Stent-Assisted Endovascular Therapy of Ruptured Intracranial Aneurysms and Relevance to Antiplatelet Administration: A Systematic Review
}

\author{
(D) C.-W. Ryu, S. Park, DH.S. Shin, and J.S. Koh
}

\begin{abstract}
BACKGROUND AND PURPOSE: Despite the increasing use of stent-assisted coiling for ruptured intracranial aneurysms, there is little consensus regarding the appropriate antiplatelet administration for this. The objectives of this systematic review were to provide an overview of complications and their association with the method of antiplatelet administration in stent-assisted coiling for ruptured intracranial aneurysms.
\end{abstract}

MATERIALS AND METHODS: A comprehensive search of the literature in the data bases was conducted to identify studies reporting complications of stent-assisted coiling for ruptured intracranial aneurysms. The pooled event rate of preprocedural thromboembolisms, hemorrhages, and mortality was estimated from the selected studies. Subgroup analyses were performed by the method of antiplatelet administration (pre-, postprocedural, and modified). Meta-analysis was conducted to compare periprocedural complications and mortality between ruptured intracranial aneurysms and unruptured intracranial aneurysms.

RESULTS: Of the 8476 studies identified, 33 with 1090 patients were included. The event rates of thromboembolism and intra- and postprocedural hemorrhage were $11.2 \%(95 \% \mathrm{Cl}, 9.2 \%-13.6 \%)$, 5.4\% (95\% Cl, 4.1\%-7.2\%), and 3.6\% (95\% Cl, 2.6\%-5.1\%), respectively. Subgroup analyses of thromboembolism showed a statistically significant difference between groups $(P<.05)$. In the preprocedural and modified antiplatelet groups, the risk for thromboembolism in stent-assisted coiling for ruptured intracranial aneurysm was not significantly different from that for unruptured intracranial aneurysm, though this risk of the postprocedural antiplatelet group was significantly higher in ruptured intracranial aneurysms than in unruptured intracranial aneurysms.

CONCLUSIONS: On the basis of current evidence, complications of stent-assisted coiling for ruptured intracranial aneurysm may be affected by the method of antiplatelet administration.

ABBREVIATIONS: RIA = ruptured intracranial aneurysm; RR = risk ratio; TEC = thromboembolic complication; UIA = unruptured intracranial aneurysm

A neurysmal neck remodeling with stents has recently emerged as an effective treatment option. This method is beneficial for treating aneurysms with wide necks or for situations in which coils unexpectedly herniate into the parent vessel, requiring rescue with a device that can reconstrain the coil within the lesion. ${ }^{1}$ Currently, various stents specialized for aneurysmal neck remodeling are used during endovascular treatment of intracranial an-

Received December 14, 2014; accepted after revision February 11, 2015.

From the Departments of Radiology (C.-W.R., S.P.) and Neurosurgery (H.S.S., J.S.K.), Kyung Hee University Hospital at Gangdong, Kyung Hee University School of Medicine; Seoul, South Korea.

Please address correspondence to Chang-Woo Ryu, MD, Department of Radiology, Kyung Hee University Hospital at Gangdong, 892 Dongnam-ro, Gangdong-gu, Seoul, 134-727, South Korea; e-mail: md.cwryu@gmail.com

Indicates article with supplemental on-line table.

Evidence-Based Medicine Level 1.

http://dx.doi.org/10.3174/ajnr.A4365 eurysms. However, physicians are often reluctant to apply stents to acutely ruptured aneurysms due to the necessity of antiplatelet medications. During implantation of stents within an intracranial artery, antiplatelet agents should be administrated and maintained postoperatively to prevent in-stent thrombosis and subsequent ischemic events. ${ }^{2}$ In the setting of acutely ruptured aneurysms, antiplatelet medications may lead to complications such as intraprocedural rebleeding, the need for a ventriculostomy, cooccurrence of an intraparenchymal hematoma, and a high likelihood of future invasive procedures. ${ }^{3-7}$

Despite the chance of complications, administration of antiplatelet agents is an important element of management when using an intracranial stent, regardless of the presence of an acute aneurysm rupture. The type and/or method of antiplatelet agent might affect the periprocedural complication rate of endovascular aneurysm treatment. ${ }^{8,9}$ Despite many previous studies of stentassisted aneurysm management, no published recommendations 
or large randomized clinical trials provide a consensus as to the appropriate method of antiplatelet medication in stent-assisted endovascular treatment for ruptured intracranial aneurysms (RIAs). The medication method usually varied depending on the institution or the rationales of clinicians in most published case series. Some review articles suggested a higher risk of complications in endovascular therapy for acutely ruptured aneurysms. ${ }^{3,10,11}$ However, these reviews did not analyze independent factors affecting the risk of complications in stent-assisted coiling for RIA, including the application of antiplatelet agents.

The purposes of this systematic review were to calculate the accumulated complication risk during stent-assisted coiling for RIA and to assess whether the risk of complications would be affected by the method of antiplatelet administration. This information will guide selection of safer antiplatelet administration for stent-assisted coiling of RIA.

\section{MATERIALS AND METHODS \\ Search Strategy}

We searched the PubMed, EMBASE, and Cochrane data bases from January 2000 to November 2014 to find all studies involving stent-assisted coiling for acute RIA. The following key words and alternative terms analogous to these were used for searching relevant combinations by using the Boolean operators OR and AND: "Intracranial Aneurysm," "Subarachnoid Hemorrhage," "Endovascular Procedures," "Stents," and "Embolization, Therapeutic." In addition, the names of merchandized stent devices, such as "Neuroform," "Enterprise," "Leo+ stent" and "Solitaire" were used as text words to extract relevant studies. We also searched the references of all included articles for potentially relevant trials. The search was restricted to human studies in English.

Two reviewers (C.-W.R., S.P.) independently evaluated the articles in the librarian's primary list and selected any articles that fulfilled the inclusion criteria as follows: 1 ) reported $>5$ cases of RIA treated with stent-assisted coiling; 2) were published as full articles, including clinical trials, retrospective or prospective case series, or retrospective observational studies; 3 ) had well-described protocols for endovascular treatment of RIA, including a precise report on each antiplatelet regimen and endovascular device; and 4) accurately described periprocedural complications, procedure-induced mortality, and/or mortality within 30 days.

When the same cases were duplicated in multiple publications, data were extracted from the publication that provided the most comprehensive information. Reports including only dissecting aneurysms and/or blood blister-like aneurysms were excluded. A PRISMA flow diagram was used to show the decision-making process regarding the studies.

\section{Data Extraction}

Basic characteristics of articles including publication type (prospective or retrospective, registry, or randomized trials), number of subjects, and publication year were extracted from each article. We extracted data relating to characteristics of enrolling patients, such as the total number of patients with RIA and unruptured intracranial aneurysm (UIA) and the initial clinical status, which was presented as Hunt and Hess grade or World Federation of Neurological Surgeons SAH grade.

We collected information about the method of administration and regimen of antiplatelets and administration of anticoagulation agents and procedural complications, including periprocedural thromboembolic complications (TECs), intraprocedural hemorrhage, postprocedural hemorrhage, clinical outcome, and periprocedural mortality (within 30 days or discharge).

For studies that did not provide the prevalence of complications in RIAs separately from those in UIAs, we e-mailed the author to obtain this information, which is included here as data.

\section{Statistical Analysis}

Pooled Event Rate of Complications. The pooled event rate of each periprocedural complication, morbidity, and mortality was estimated with a random-effects-weighting meta-analysis. Because the definition of "morbidity" varied in each study, it was defined as mRS 3-5 or Glasgow Outcome Score 2-3 at discharge or follow-up of $\geq 3$ months. The corresponding $95 \%$ CIs for single proportions were determined with the binomial theorem. Heterogeneity across studies in the meta-analysis was assessed by using the $\mathrm{I}^{2}$ test, in which $>40 \%$ is considered substantial heterogeneity. Sources of heterogeneity were appraised by subgroup stratification analysis based on the method of antiplatelet therapy. Between-group heterogeneity values were calculated to assess the variance of the distribution of the true effect sizes among subgroups, and a $P$ value of $<.05$ was considered statistically significant.

Risk Ratio of Periprocedural Complications of Stent-Assisted Coiling in RIA Relative to UIA. To evaluate the difference in complication rates of stent-assisted coiling between RIA and UIA, we selected studies containing data from both RIAs and UIAs from studies including previous event-rate analyses. Studies containing $<10$ cases in each group or studies in which antiplatelets were administered after the procedure in stent-assisted coiling for UIA were excluded from the review.

Meta-analysis was conducted to compare periprocedural complications and procedure-induced mortality between RIA and UIA groups. Meta-analysis was performed by using Review Manager (Version 5.3; http://review-manager.software. informer.com/5.3/). Complications and mortality were reported with a risk ratio (RR) and forest plot by using Mantel Haenszel random-effects models. Heterogeneity across studies in the metaanalysis was assessed by using the $\mathrm{I}^{2}$ test. Subgroup analyses also were performed by the method of antiplatelet administration.

\section{RESULTS}

\section{Study Characteristics}

A total of 8476 studies were identified during the initial search. Of these, 33 studies with 1090 participants were pertinent. ${ }^{7,9,12-42}$ Figure 1 is the flow diagram summarizing the literature search results. There were no randomized clinical trials in the selected studies. Three studies were prospective registry studies, and 30 were retrospective observational studies. Nineteen studies included subjects who received stent-assisted coiling for both UIA 


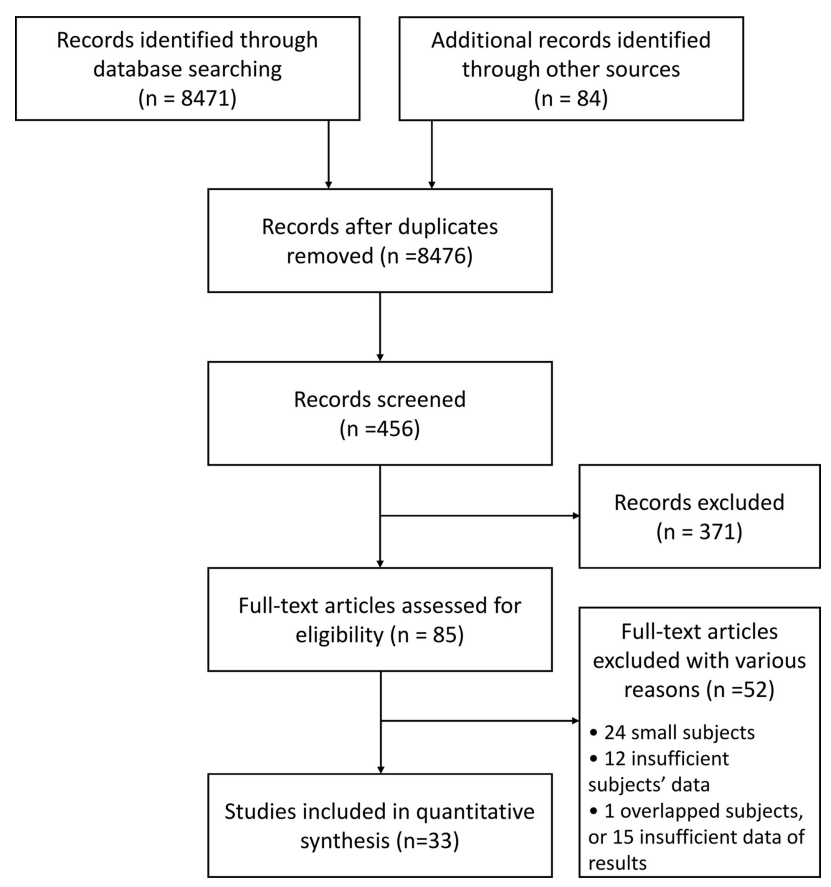

FIG 1. PRISMA flow diagram summarizing the search and selection of articles.

and RIA, while 14 studies included only RIA cases (On-line Table).

\section{Characteristics of Cohorts, Antiplatelet Method, and Endovascular Procedures}

Initial clinical status could be extracted from 726 patients in 22 studies. Five hundred fifty-one patients $(75.9 \%)$ had good initial clinical grades (Hunt and Hess or World Federation of Neurological Surgeons grades 1-3), and 175 patients (24.1\%) had bad initial clinical grades (Hunt and Hess or World Federation of Neurological Surgeons grades 4 and 5).

In all 33 studies, antiplatelet therapy for stent-assisted coiling of RIA followed the preset protocol identified in each study. Eleven studies (331 patients) administrated a loading dose of classic dual antiplatelet agents (aspirin and clopidogrel) at least 2 hours before the start of the procedure. Seventeen studies (560 patients) administered a loading dose of dual antiplatelet agents after completion of the endovascular procedure or postprocedural dual antiplatelet agents with intraprocedural administration of a kind of antiplatelet agent. The other 5 studies (199 patients) used the modified method by using intravenous administration of glycoprotein IIb/IIIa inhibitor (eptifibatide in 3 and tirofiban in 2 studies) after stent deployment. In all studies, anticoagulation treatments with heparin were maintained during the endovascular procedure, except the cases with extravasation on the intraprocedural angiogram. We classified the enrolled studies into 3 groups based on different methods of antiplatelet administration: the preprocedural, postprocedural, and modified antiplatelet administration group.

\section{Pooled Incidence of Periprocedural Complications}

TEC and intra- and postprocedural hemorrhage rates were available in all 33 enrolled studies. Periprocedural TECs occurred in
108 patients (event rate: $11.2 \%$; 95\% CI, 9.2\%-13.6\%; $\mathrm{I}^{2}=1 \%$ ). In the subgroup analysis, the event rates of TEC in preprocedural, postprocedural, and modified antiplatelet groups were $7.4 \%$ (95\% CI, 5.0\%-11.0\%; $\mathrm{I}^{2}=0 \%$ ), $12.3 .0 \%$ (95\% CI, 9.2\%-16.4\%; $\left.\mathrm{I}^{2}=21 \%\right)$, and $13.9 \%\left(95 \% \mathrm{CI}, 9.7 \%-19.5 \% ; \mathrm{I}^{2}=0 \%\right)$, respectively. The between-group heterogeneity was significant $(P=$ .046). An intraprocedural hemorrhage occurred in 46 patients (event rate: $5.4 \%$; 95\% CI, 4.1\%-7.2\%; $\mathrm{I}^{2}=0 \%$ ), and event rates in the preprocedural, postprocedural, and modified antiplatelet groups were 5.3\% (95\% CI, 3.3\%-8.5\%; $\left.\mathrm{I}^{2}=0 \%\right), 4.9 \%$ (95\% CI, $3.3 \%-7.4 \%$; $\left.\mathrm{I}^{2}=0 \%\right)$, and $8.8 \%\left(5.2 \%-14.4 \% ; \mathrm{I}^{2}=29 \%\right)$. The overall event rate of postprocedural hemorrhage was 3.6\% (95\% CI, $2.6 \%-5.1 \%$; $\mathrm{I}^{2}=0 \%$ ), and subgroup event rates of preprocedural, postprocedural, and modified antiplatelet groups were 2.9\% (95\% CI, 1.4\%-5.9\%; $\mathrm{I}^{2}=0 \%$ ), 3.9\% (95\% CI, 2.5\%-6.3\%; $\left.\mathrm{I}^{2}=0 \%\right)$, and $3.7 \%\left(1.7 \%-7.9 \% ; \mathrm{I}^{2}=0 \%\right)$. There was no significant difference between groups in these 2 hemorrhagic complications.

Morbidity was extracted from 22 studies, and it was measured at 3-6 months in 5 studies, at discharge in 11 studies, and at an unspecified time (such as "last follow-up") in 6 studies. Mortality was extracted from 28 studies. Pooling of morbidity and mortality yielded substantial heterogeneity $\left(\mathrm{I}^{2}=54 \%\right.$ and $56 \%)$. In the subgroup analysis, substantial heterogeneity was shown only in the preprocedural antiplatelet groups, and the event rate of morbidity in this subgroup was $9.3 \%$ (95\% CI, $6.2 \%-13.7 \%$; $\left.\mathrm{I}^{2}=0 \%\right)$. Ranges of incidences of morbidity in the postprocedural and modified antiplatelet group were $3.1 \%-49.2 \%$ and $2.9 \%-20.0 \%$. The pooling mortality rates of pre- and postprocedural antiplatelet groups were 9.8\% (95\% CI, 5.7\%-16.4\%; $\mathrm{I}^{2}=37 \%$ ) and $13.4 \%$ (95\% CI, 9.3\%-19.0\%; $\left.\mathrm{I}^{2}=38 \%\right)$. The mortality range of the modified antiplatelet group was $4.3 \%-36.0 \%$. Although significant between-group heterogeneity was present in both morbidity and mortality, these findings were ignored due to the heterogeneity within each subgroup.

\section{Risk Ratio of Periprocedural Complications of Stent- Assisted Coiling in RIA Relative to UIA}

Thirteen studies were enrolled in this analysis to assess the risk ratio of periprocedural complications of stent-assisted coiling in RIA compared with UIA. There were 505 patients in the RIA group and 1790 patients in the UIA group. These studies were composed of 5 preprocedural, 5 postprocedural, and 3 modified antiplatelet groups. We did not address the meta-analysis regarding postprocedural hemorrhage and morbidity because of too many inestimable studies.

TEC occurred more frequently in patients with RIAs with stent-assisted coiling than in patients with UIAs with stentassisted coiling ( $\left.\mathrm{RR}, 2.25 ; 95 \% \mathrm{CI}, 1.43-3.55 ; \mathrm{I}^{2}=33 \%\right)$. The risk of intraprocedural hemorrhage ( $\mathrm{RR}, 5.03 ; 2.42-10.45 ; \mathrm{I}^{2}=$ $0 \%$ ) and mortality (RR, 7.39; 95\% CI, 3.40-16.03; $\mathrm{I}^{2}=39 \%$ ) was also higher in the RIA than in the UIA group (Fig 2).

In the preprocedural and modified antiplatelet groups, the TEC risk in RIA was not significantly different from that in UIA (RR, 1.34; 95\% CI, 0.59-3.06; $\mathrm{I}^{2}=0 \%$; and RR, 1.65; 95\% CI, $\left.0.96-2.85 ; \mathrm{I}^{2}=0 \%\right)$. However, TEC occurred more frequently in 
Risk Ratio

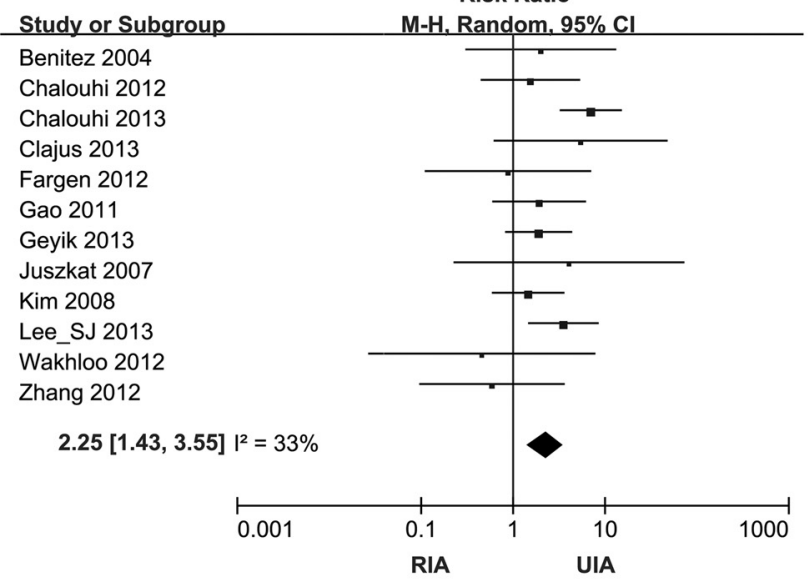

Risk Ratio

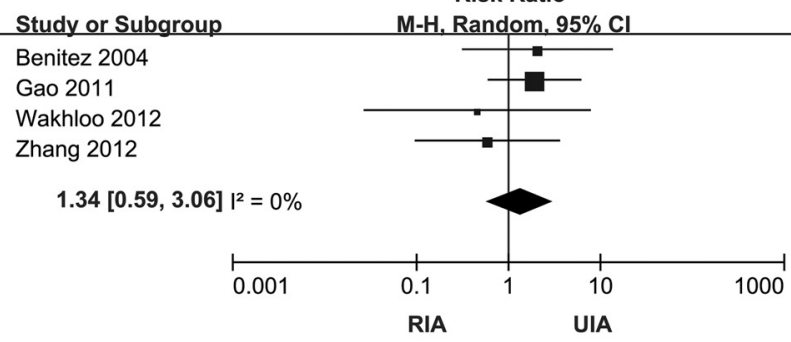

Risk Ratio

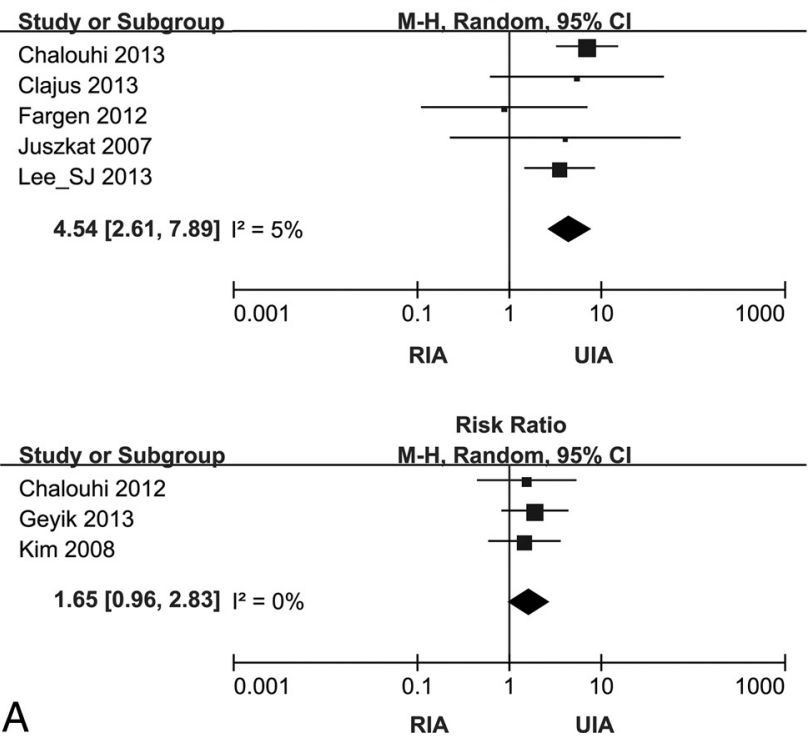

RIA than in UIA in the postprocedural antiplatelet group (RR, 4.54; 95\% CI, 2.61-7.89; $\mathrm{I}^{2}=5 \%$ ) (Fig 2A). Subgroup analysis for intraprocedural hemorrhage showed that the postprocedural antiplatelet group had significantly higher rates (RR, 6.68; 95\% CI, 2.31-19.36; $\mathrm{I}^{2}=0 \%$ ) in RIA compared with UIA (Fig $2 B$ ). Subgroup analysis for intraprocedural hemorrhage of the preprocedural antiplatelet group yielded substantial heterogeneity $\left(\mathrm{I}^{2}=\right.$ $54 \%)$. Subgroup analysis for mortality showed that it was significant higher in RIA relative to UIA in both pre- and postprocedural antiplatelet groups (RR, 7.64; 95\% CI, 1.95-29.94; $\mathrm{I}^{2}=$ 28\%; and RR, 13.44; 95\% CI, 5.14-35.17; $\mathrm{I}^{2}=0 \%$ ) (Fig 2C). Because results of the meta-analyses for modified-antiplatelet groups showed heterogeneity $\left(\mathrm{I}^{2}=76 \%\right)$ in intraprocedural hem-

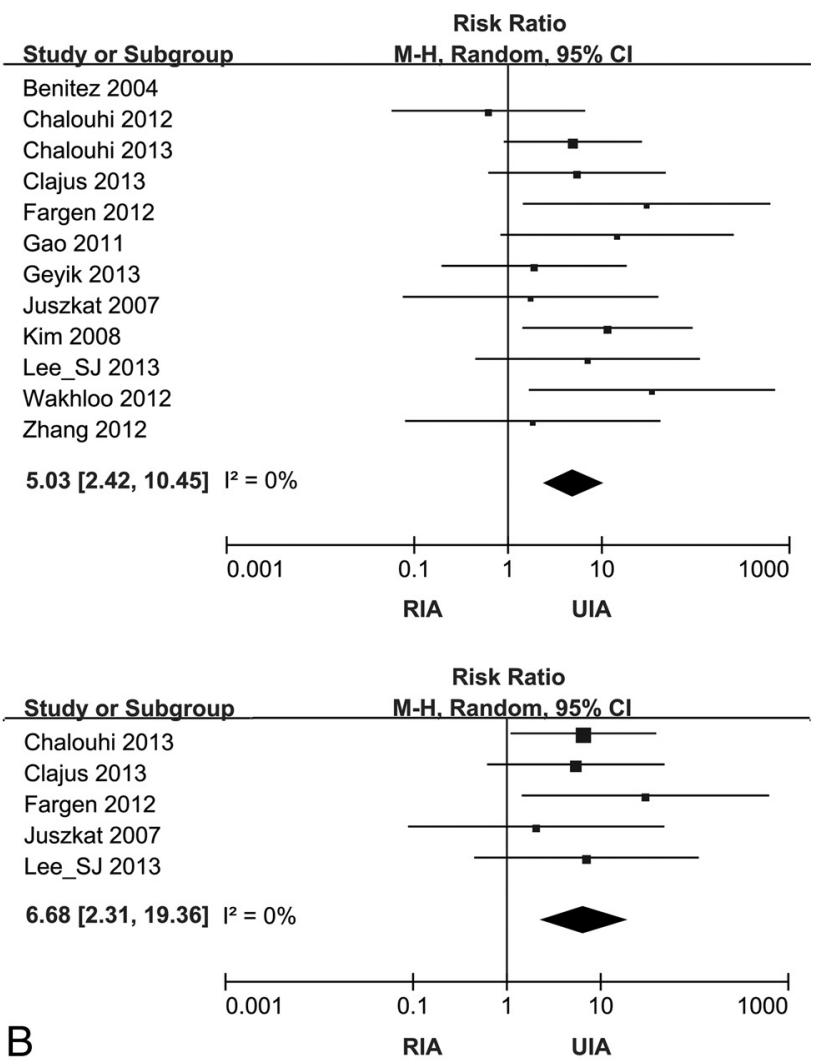

FIG 2. Forest plots showing the risk ratio for complications and the mortality of stent-assisted coiling comparing RIA and UIA. Effect sizes are plotted with $95 \% \mathrm{Cls}$. A, Forest plots for TEC. From uppermost to lowest, plots for the pooled studies and preprocedural, postprocedural, and modified antiplatelet subgroups. B, Forest plots for intraprocedural hemorrhage. The upper plot is for pooled studies, and lower one is for the postprocedural antiplatelet subgroup. $C$ (next page), Forest plots for mortality. Starting with the uppermost, plots for the pooled studies and pre- and postprocedural antiplatelet subgroups.

orrhage and mortality, we did not present these with forest plots.

\section{DISCUSSION}

In terms of endovascular treatment of RIA, many clinicians were concerned about the potential risk of the neck-remodeling technique, such as the complexity of the procedure, thrombotic risk, and antithrombotic drugs. Several studies such as CLARITY (Clinical and Anatomic Results in the Treatment of Ruptured Intracranial Aneurysms) reported that in treatment of ruptured aneurysms, the remodeling technique was as safe as conventional coil embolization and had higher rates of ad- 
Risk Ratio
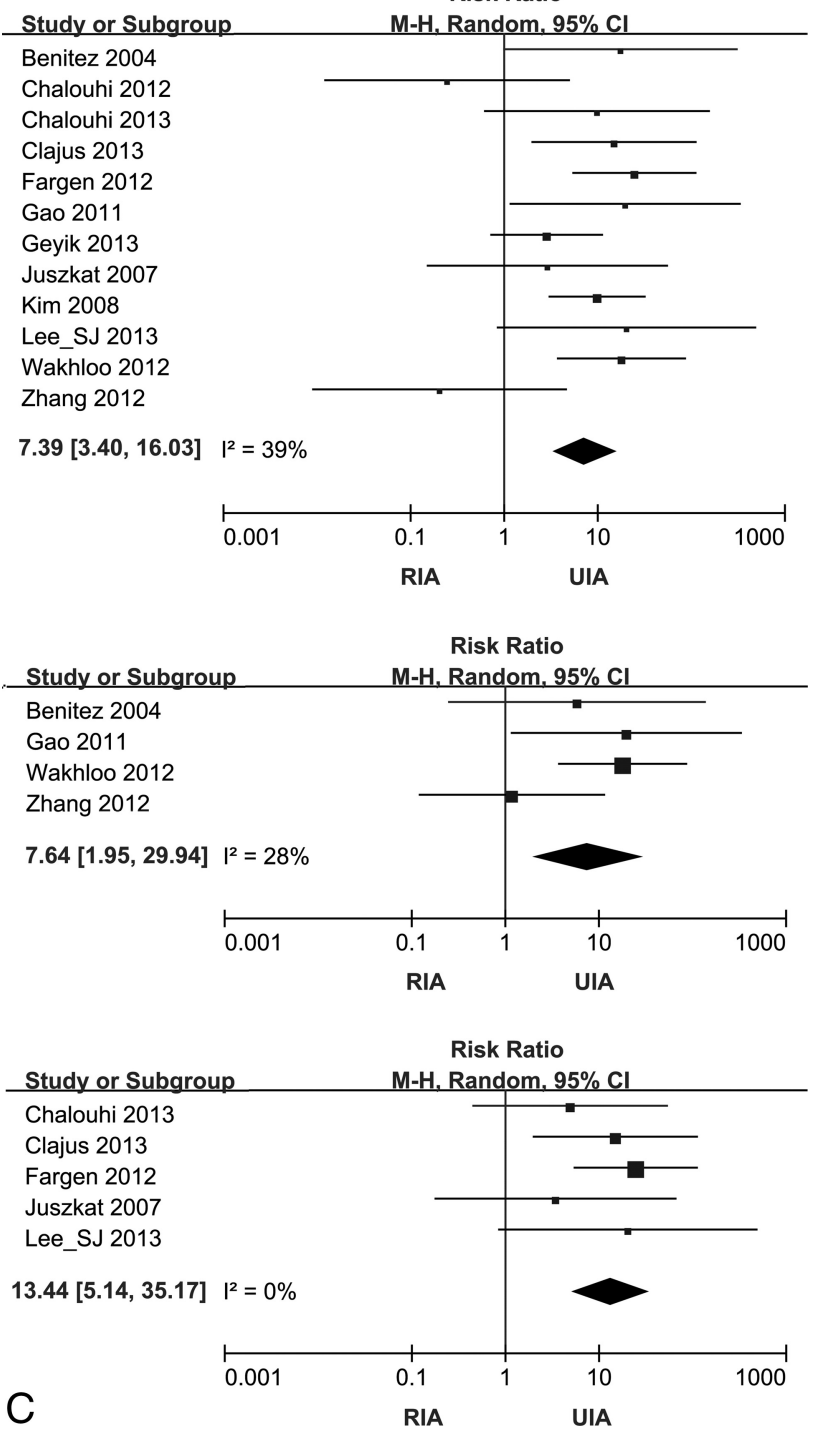

FIG 2. Continued

equate postoperative occlusion. ${ }^{43}$ However, this assertion did not have enough evidence to end the concern about the risk of stent-assisted coiling in ruptured aneurysms. In this systematic review, the pooled incidence of TEC and periprocedural hemorrhages in stent-assisted coiling of RIA varied from $3.6 \%$ to $11.3 \%$, which was higher than the complication rates that have been previously reported in large randomized trials of the coil embolization of RIA. ${ }^{44,45}$

Despite the present review, stent-assisted coiling for UIA is now accepted as a safe treatment option. Several studies demonstrated that the complication risk of stent-assisted coiling was not significantly higher than that in coil-alone treatment, though stented aneurysms usually have more difficult morphology than coil-alone aneurysms. ${ }^{46-49}$ Most important, the stent-assisted coiling has a high rate of adequate occlusion, leading to a decrease in the recanalization rate in mid- or long-term follow-up. ${ }^{48,50}$ The situation of acutely ruptured aneurysms makes it difficult to guarantee that the safety of stent-assisted coiling is the same as that for coiling alone, and research should be focused on finding more safe ways to implement stent-assisted coiling in RIA. In this re- gard, antiplatelet medication use during endovascular RIA management is of considerable interest to clinicians because it can elevate the risk of hemorrhagic complications while preventing TEC. Nevertheless, this review showed that there was little consensus about the safe timing of antiplatelet administration that minimizes the complications in stent-assisted coiling of RIA. The methods of antiplatelet therapy varied depending on the institutions, and no high-quality evidence supports the various methods.

The result of subgroup analysis showed that the event rate of TEC varied among the different methods of antiplatelet administration. These findings support the presumption that the complications in stent-assisted RIA can be affected by the method of antiplatelet administration. That stent-assisted coiling for RIA has higher complication and mortality rates than for UIA is easily predictable. This meta-analysis also showed that the pooled risk of complication and mortality rates was significantly higher in RIA than UIA. However, in the preprocedural antiplatelet subgroup, the risk of TEC was not different between RIA and UIA. These results were contrary to the higher prevalence of RIA compared with UIA in the postprocedural antiplatelet analysis and suggested that preprocedural administration of antiplatelets might reduce the risk of TECs during stent-assisted coiling of RIA. Unfortunately, this study could not reveal which antiplatelet method could reduce the risk of hemorrhagic complications.

The present study has several limitations. First, this review was unable to include a well-designed case-control prospective study; we could not find enough studies that compared different antiplatelet therapies or complication risks between stent-assisted coiling and simple coiling for RIA to get statistically significant results. Therefore, our inferences were indirectly suggested from comparisons between the stent-assisted coiling of RIA and UIA. Second, although the severity of morbidity can vary depending on the type of complications, this analysis did not address the impact of the associated surgical procedures that complicate the risk of hemorrhage and hemorrhagerelated morbidity that may result from antiplatelet use. Finally, this review also has the common limitations that threaten the validity of systematic reviews, such as publication bias and the generalization of results from different reviews. The retrospective nature of most included studies affected the availability of some variables in some studies and resulted in the absence of standardized definitions for available variables.

\section{CONCLUSIONS}

Although various methods of antiplatelet administration have been used in published articles, there is little consensus regarding the appropriate and safe timing for the administration of antiplatelet agents. However, this study revealed that the clinical results of stentassisted coiling for RIA would be affected by antiplatelet administration. Therefore, reliable guidelines for antiplatelet therapy in stentassisted coiling for RIA should be determined by the results of future research. The results of this systematic review can guide future prospective case-control study design to identify more appropriate antiplatelet therapy in stent-assisted coiling of RIA. 


\section{REFERENCES}

1. Yoo E, Kim DJ, Kim DI, et al. Bailout stent deployment during coil embolization of intracranial aneurysms. AJNR Am J Neuroradiol 2009;30:1028-34

2. Hwang G, Kim JG, Song KS, et al. Delayed ischemic stroke after stent-assisted coil placement in cerebral aneurysm: characteristics and optimal duration of preventative dual antiplatelet therapy. $R a-$ diology 2014;273:194-201

3. Bodily KD, Cloft HJ, Lanzino G, et al. Stent-assisted coiling in acutely ruptured intracranial aneurysms: a qualitative, systematic review of the literature. AJNR Am J Neuroradiol 2011; 32:1232-36

4. Zhang XD, Wu HT, Zhu J, et al. Delayed intracranial hemorrhage associated with antiplatelet therapy in stent-assisted coil embolized cerebral aneurysms. Acta Neurochir Suppl 2011;110(pt 2):133-39

5. Manabe H. Ruptured cerebral aneurysms treated by stent-assisted GDC embolization-two case reports with long-term follow-up. Acta Neurochir Suppl 2008;103:5-8

6. Tumialán LM, Zhang YJ, Cawley CM, et al. Intracranial hemorrhage associated with stent-assisted coil embolization of cerebral aneurysms: a cautionary report. J Neurosurg 2008;108:1122-29

7. Taylor RA, Callison RC, Martin CO, et al. Acutely ruptured intracranial saccular aneurysms treated with stent assisted coiling: complications and outcomes in $\mathbf{4 2}$ consecutive patients. J Neurointerv Surg 2010;2:23-30

8. Ries T, Buhk JH, Kucinski T, et al. Intravenous administration of acetylsalicylic acid during endovascular treatment of cerebral aneurysms reduces the rate of thromboembolic events. Stroke 2006;37:1816-21

9. Chalouhi N, Jabbour P, Kung D, et al. Safety and efficacy of tirofiban in stent-assisted coil embolization of intracranial aneurysms. $\mathrm{Neu}$ rosurgery 2012;71:710-14; discussion 714

10. Ferns SP, Sprengers ME, van Rooij WJ, et al. Coiling of intracranial aneurysms: a systematic review on initial occlusion and reopening and retreatment rates. Stroke 2009;40:e523-529

11. Naggara ON, White PM, Guilbert F, et al. Endovascular treatment of intracranial unruptured aneurysms: systematic review and metaanalysis of the literature on safety and efficacy. Radiology 2010;256:887-97

12. Amenta PS, Dalyai RT, Kung D, et al. Stent-assisted coiling of wide-necked aneurysms in the setting of acute subarachnoid hemorrhage: experience in 65 patients. Neurosurgery 2012;70:141529; discussion 1429

13. Benitez RP, Silva MT, Klem J, et al. Endovascular occlusion of widenecked aneurysms with a new intracranial microstent (Neuroform) and detachable coils. Neurosurgery 2004;54:1359-67; discussion 1368

14. Biondi A, Janardhan V, Katz JM, et al. Neuroform stent-assisted coil embolization of wide-neck intracranial aneurysms: strategies in stent deployment and midterm follow-up. Neurosurgery 2007;61: 460-68; discussion 468-69

15. Chalouhi N, Drueding R, Starke RM, et al. In-stent stenosis after stent-assisted coiling: incidence, predictors and clinical outcomes of 435 cases. Neurosurgery 2013;72:390-96

16. Clajus C, Sychra V, Strasilla C, et al. Stent-assisted coil embolization of intracranial aneurysms using the Solitaire $\mathrm{AB}$ neurovascular remodeling device: initial and midterm follow-up results. Neuroradiology 2013;55:629-38

17. Fargen KM, Hoh BL, Welch BG, et al. Long-term results of Enterprise stent-assisted coiling of cerebral aneurysms. Neurosurgery 2012;71:239-44; discussion 244

18. Galal A, Bahrassa F, Dalfino JC, et al. Stent-assisted treatment of unruptured and ruptured intracranial aneurysms: clinical and angiographic outcome. Br J Neurosurg 2013;27:607-16

19. Gao X, Liang G, Li Z, et al. Complications and adverse events associated with Neuroform stent-assisted coiling of wide-neck intracranial aneurysms. Neurol Res 2011;33:841-52

20. Huang Q, Xu Y, Hong B, et al. Stent-assisted embolization of wide- neck anterior communicating artery aneurysms: review of 21 consecutive cases. AJNR Am J Neuroradiol 2009;30:1502-06

21. Jankowitz B, Thomas AJ, Vora N, et al. Risk of hemorrhage in combined Neuroform stenting and coil embolization of acutely ruptured intracranial aneurysms. Interv Neuroradiol 2008;14: 385-96

22. Juszkat R, Nowak S, Smól S, et al. Leo stent for endovascular treatment of broad-necked and fusiform intracranial aneurysms. Interv Neuroradiol 2007;13:255-69

23. Katsaridis V, Papagiannaki C, Violaris C. Embolization of acutely ruptured and unruptured wide-necked cerebral aneurysms using the Neuroform2 stent without pretreatment with antiplatelets: a single center experience. AJNR Am J Neuroradiol 2006;27:1123-28

24. Kim SR, Vora N, Jovin TG, et al. Anatomic results and complications of stent-assisted coil embolization of intracranial aneurysms. Interv Neuroradiol 2008;14:267-84

25. Kim YJ. Early experiences of Neuroform stent-assisted coiling in ruptured intracranial aneurysms. Interv Neuroradiol 2007;13: 31-44

26. Klisch J, Eger C, Sychra V, et al. Stent-assisted coil embolization of posterior circulation aneurysms using Solitaire AB: preliminary experience. Neurosurgery 2009;65:258-66; discussion 266

27. Lee SJ, Cho YD, Kang HS, et al. Coil embolization using the selfexpandable closed-cell stent for intracranial saccular aneurysm: a single-center experience of $\mathbf{2 8 9}$ consecutive aneurysms. Clin Radiol 2013;68:256-63

28. Lodi YM, Latorre JG, El-Zammar Z, et al. Stent assisted coiling of the ruptured wide necked intracranial aneurysm. J Neurointerv Surg 2012;4:281-86

29. Piotin M, Blanc R, Spelle L, et al. Stent-assisted coiling of intracranial aneurysms: clinical and angiographic results in 216 consecutive aneurysms. Stroke 2010;41:110-15

30. Sedat J, Chau Y, Mondot L, et al. Endovascular occlusion of intracrania wide-necked aneurysms with stenting (Neuroform) and coiling: midterm and long-term results. Neuroradiology 2009;51:401-09

31. Tähtinen OI, Vanninen RL, Manninen HI, et al. Wide-necked intracranial aneurysms: treatment with stent-assisted coil embolization during acute ( $<72$ hours) subarachnoid hemorrhage-experience in 61 consecutive patients. Radiology 2009;253:199-208

32. Wakhloo AK, Linfante I, Silva CF, et al. Closed-cell stent for coil embolization of intracranial aneurysms: clinical and angiographic results. AJNR Am J Neuroradiol 2012;33:1651-56

33. Zhang J, Lv X, Yang J, et al. Stent-assisted coil embolization of intracranial aneurysms using Solitaire stent. Neurol India 2012;60:278-82

34. Yun JH, Cho CS. Experiences of Neuroform stent applications for ruptured anterior communicating artery aneurysms with small parent vessel. J Korean Neurosurg Soc 2010;48:53-58

35. Chung J, Lim YC, Suh SH, et al. Stent-assisted coil embolization of ruptured wide-necked aneurysms in the acute period: incidence of and risk factors for periprocedural complications. J Neurosurg 2014;121:4-11

36. Geyik S, Yavuz K, Yurttutan N, et al. Stent-assisted coiling in endovascular treatment of $\mathbf{5 0 0}$ consecutive cerebral aneurysms with long-term follow-up. AJNR Am J Neuroradiol 2013;34:2157-62

37. Huang QH, Wu YF, Shen J, et al. Endovascular treatment of acutely ruptured, wide-necked anterior communicating artery aneurysms using the Enterprise stent. J Clin Neurosci 2013;20:267-71

38. Zhao R, Shen J, Huang QH, et al. Endovascular treatment of ruptured tiny, wide-necked posterior communicating artery aneurysms using a modified stent-assisted coiling technique. J Clin $\mathrm{Neu}$ rosci 2013;20:1377-81

39. Li C, Li Y. Stent-assisted coiling of ruptured wide-necked intracranial aneurysms. Interv Neuroradiol 2013;19:283-88

40. Yang P, Liu J, Huang Q, et al. Endovascular treatment of wide-neck middle cerebral artery aneurysms with stents: a review of 16 cases. AJNR Am J Neuroradiol 2010;31:940-46

41. Chitale R, Chalouhi N, Theofanis T, et al. Treatment of ruptured 
intracranial aneurysms: comparison of stenting and balloon remodeling. Neurosurgery 2013;72:953-59

42. Lee WJ, Cho CS. Y-stenting endovascular treatment for ruptured intracranial aneurysms: a single-institution experience in Korea. $J$ Korean Neurosurg Soc 2012;52:187-92

43. Pierot L, Cognard C, Anxionnat R, et al; CLARITY Investigators. Remodeling technique for endovascular treatment of ruptured intracranial aneurysms had a higher rate of adequate postoperative occlusion than did conventional coil embolization with comparable safety. Radiology 2011;258:546-53

44. Pierot L, Cognard C, Anxionnat R, et al; CLARITY Investigators. Ruptured intracranial aneurysms: factors affecting the rate and outcome of endovascular treatment complications in a series of 782 patients (CLARITY study). Radiology 2010;256:916-23

45. Molyneux AJ, Kerr RS, Yu LM, et al; International Subarachnoid Aneurysm Trial (ISAT) Collaborative Group. International Subarachnoid Aneurysm Trial (ISAT) of neurosurgical clipping versus endovascular coiling in 2143 patients with ruptured intracranial aneurysms: a randomised comparison of effects on survival, dependency, seizures, re- bleeding, subgroups, and aneurysm occlusion. Lancet 2005;366: 809-17

46. Frontera JA, Moatti J, de los Reyes KM, et al. Safety and cost of stentassisted coiling of unruptured intracranial aneurysms compared with coiling or clipping. J Neurointerv Surg 2014;6:65-71

47. Hetts SW, Turk A, English JD, et al; Matrix and Platinum Science Trial Investigators. Stent-assisted coiling versus coiling alone in unruptured intracranial aneurysms in the Matrix and Platinum Science trial: safety, efficacy, and mid-term outcomes. AJNR Am J Neuroradiol 2014;35:698-705

48. Hong Y, Wang YJ, Deng Z, et al. Stent-assisted coiling versus coiling in treatment of intracranial aneurysm: a systematic review and meta-analysis. PLoS One 2014;9:e82311

49. Lopes DK, Johnson AK, Kellogg RG, et al. Long-term radiographic results of stent-assisted embolization of cerebral aneurysms. $\mathrm{Neu}$ rosurgery 2014;74:286-91

50. Mine B, Aljishi A, D'Harcour JB, et al. Stent-assisted coiling of unruptured intracranial aneurysms: long-term follow-up in 164 patients with 183 aneurysms. J Neuroradiol 2014;41:322-28 